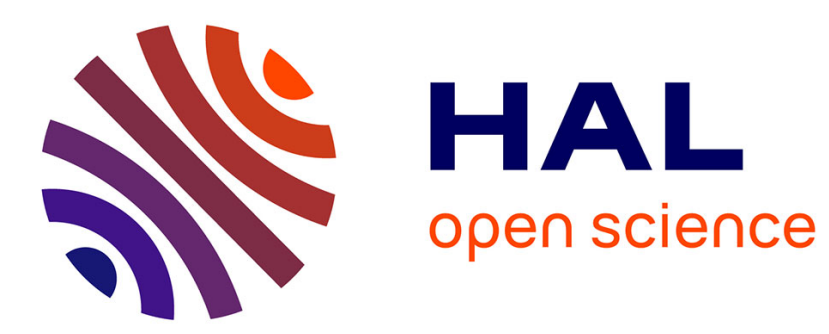

\title{
Kinetic dendrite in the boundary-layer model
}

\author{
D. Temkin, J. Géminard, P. Oswald
}

\section{To cite this version:}

D. Temkin, J. Géminard, P. Oswald. Kinetic dendrite in the boundary-layer model. Journal de Physique I, 1994, 4 (3), pp.403-409. 10.1051/jp1:1994147 . jpa-00246916

\section{HAL Id: jpa-00246916 https://hal.science/jpa-00246916}

Submitted on 1 Jan 1994

HAL is a multi-disciplinary open access archive for the deposit and dissemination of scientific research documents, whether they are published or not. The documents may come from teaching and research institutions in France or abroad, or from public or private research centers.
L'archive ouverte pluridisciplinaire HAL, est destinée au dépôt et à la diffusion de documents scientifiques de niveau recherche, publiés ou non, émanant des établissements d'enseignement et de recherche français ou étrangers, des laboratoires publics ou privés. 
Classification

Physics Abstracts

$61.50 \mathrm{C}-61.50 \mathrm{~J}-81.30 \mathrm{~F}$

\title{
Kinetic dendrite in the boundary-layer model
}

\author{
D. Temkin $\left({ }^{*}\right)$, J. C. Géminard and P. Oswald \\ Ecole Normale Supérieure de Lyon, Laboratoire de Physique, 46 Allée d'Italie, 69364 Lyon \\ Cedex 07, France
}

(Recelved 29 September 1993, accepted 3 December 1993)

\begin{abstract}
Within the boundary-layer model and by taking into account only interface kinetics (isotropic or anisotropıc), we find a contmuous set of « kinetic » dendrites. This solution exists at arbitrary supercooling $\Delta$ in the 2D-and 3D-axisymmetric cases and reduces to the usual dendrites with parabolic tails at $\Delta<1$, and « angular » dendrites at $\Delta>1$. In contrast with classical Ivantsov dendrites, there are upper limits for growth velocity and tip curvature at each supercooling $\Delta$.
\end{abstract}

Ivantsov [1] was the first to show that there exists a continuous set of stationary needle-like solutions to the problem of the free growth of a crystal. These solutions are obtained by neglecting capillarity and interface kinetics and exist only at dimensionless supercooling $\Delta$ smaller than 1. To find a single dendrite (experimentally, only one is observed), one needs to introduce a length in the problem. Usually, the capillary length $d_{c}$ is introduced and the solution is found by disturbing the Ivantsov parabolic solution using a singular perturbation method. In this case, and in two dimensions, the tip velocity $v$ is proportional to $\left(D / d_{c}\right) \Delta^{4}$ at $\Delta \ll 1$, where the constant of proportionality depends only on the anisotropy of the surface tension [2]. $D$ is the diffusion coefficient.

This choice of the length is not unique because there is another characteristic length connected with interface kinetics : that 1 , $D / w$ where $w$ is constructed from the interfacial kinetic coefficient $\beta$. The ratio $d_{\mathrm{k}}=d_{\mathrm{c}} w / D$ of these two lengths determines which of them is more important. In pure materials, $D$ is the thermal diffusivity in the liquid, whereas in alloys it is the solute diffusion coefficient in the liquid. If $d_{\mathrm{k}} \gg 1$, one expects capillarity to be more important than kinetıcs. In the opposite case, $d_{\mathrm{k}} \ll 1$, kinetics should dominate surface tension effects. The parameter $d_{\mathrm{k}}$ is a material constant which can be evaluated. For example, $d_{\mathrm{k}} \approx 0.1$ in pure materials as $\mathrm{Ni}$ (by taking $\beta=160 \mathrm{~cm} / \mathrm{s} / \mathrm{K}$ [3]) or succinonitrile (with $\beta=20 \mathrm{~cm} / \mathrm{s} / \mathrm{K}$ [4]). In contrast, $d_{\mathrm{k}}$ is usually much larger than 1 in alloys because the chemical diffusion coefficient is much smaller than the thermal diffusivity. Nevertheless,

(*) Permanent address: I. P. Bardin Institute for Ferrous Metals, 2 Baumanskaya Str. 9/23, 107005 Moscow. Russia. 
$d_{\mathrm{k}}$ can also be small in impure materials such as discotic columnar liquid crystals. For instance, we measured previously in C8HET $\beta \approx 1.3 \times 10^{-2} \mathrm{~cm} / \mathrm{s} / \mathrm{K}$ which gives $d_{\mathrm{h}} \approx 0.5$ [5]. In all these examples we thus expect kinetics to be significant even at small supercooling. For these reasons, we found it interesting to study the case $d_{k}=0$ corresponding to zero surface tension. This problem has already been discussed by Lemieux et al. [6] and by Brener et al. [7]. Both calculations are carried out using a full non-local approach and start from the Ivantsov solution. This requires that kinetic undercooling is small. Lemieux $e t$ al. found analytically for isotropic kinetics a contınuous family of solutions in the limit of small $\Delta$, while Brener et al. found a discrete set of solutions which are selected by kinetic anisotropy for $\Delta<1$. In this article, we analyze this problem for arbitrary supercooling $\Delta$ and arbitrary kinetic undercooling. We use the boundary-layer model (BLM) which has been shown to give qualitatively correct results in many situations [8]. Another important point is that we do not use Ivantsov's solution as the starting approximation.

In the two-dimensional one-sided model (no diffusion in the solid) the BLM equation for 1sothermal and steady-state solidification of a dilute alloy reads :

$$
\begin{aligned}
k_{\mathrm{E}}^{2} V(1-U) \cos \theta-\frac{K U^{2}}{\left[1+U\left(1 / k_{\mathrm{E}}-1\right)\right]} & -K \sin \theta \frac{\mathrm{d}}{\mathrm{d} \theta}\left(\frac{U^{2}}{\cos \theta\left[1+U\left(1 / k_{\mathrm{E}}-1\right)\right]}\right) \\
& +\frac{K}{V} \frac{\mathrm{d}}{\mathrm{d} \theta}\left(\frac{K U}{\cos \theta\left[1+U\left(1 / k_{\mathrm{E}}-1\right)\right]} \frac{\mathrm{d} U}{\mathrm{~d} \theta}\right)=0
\end{aligned}
$$

where $V=v / w$ and $K=\nVdash D / w$ are respectively the dimensionless velocity in the $z$-direction and the curvature of the interface. The angle between the $z$-axis and the normal to the front $1 \mathrm{~s}$ $\theta$ and $K$ is the curvature defined by $K=\mathrm{d} \theta / \mathrm{d} s$ where $s$ is the arclength along the interface measured in units of $D / w$. The dimensionless undercooling at the interface $U$ is defined to be $U=\left(C_{\text {int }}-C_{\infty}\right) /\left[C_{\infty}\left(1 / k_{\mathrm{E}}-1\right)\right]$ where $C_{\text {int }}$ and $C_{\infty}$ are concentrations of solute at the interface and in the liquid far from the interface $; k_{\mathrm{E}}$ is the equilibrium distribution coefficient.

Equation (1) is still valid for pure materials and thermal growth. In this case, $U=\left(T_{\mathrm{int}}-T_{\infty}\right) /(L / C)$ where $L$ is the latent heat per unit volume and $c$ the heat capacity. $k_{\mathrm{E}}$ must also be set equal to 1 in equation (1) which gives the same equation as that previously used by Langer and Hong [9].

The second important equation comes from the Gibbs-Thomson relation. Without anisotropy effects, it is

$$
U=\Delta-d_{\mathrm{k}} K-V \cos \theta
$$

w1th $d_{\mathrm{k}}=d_{\mathrm{c}} w / D$. In the chemical case, $\Delta=\left(C_{\mathrm{LE}}-C_{\infty}\right) /\left[C_{\infty}\left(1 / k_{\mathrm{E}}-1\right)\right]$ where $C_{\mathrm{LE}}$ is the equilibrium solute concentration in the liquid at the temperature chosen. The capillary length is $d_{\mathrm{c}}=\left(\gamma T_{\mathrm{m}} / L\right) /\left[m C_{\infty}\left(1 / k_{\mathrm{E}}-1\right)\right]$ and $w=\beta m C_{\infty}\left(1 / k_{\mathrm{E}}-1\right)$ where $\gamma$ is the surface stiffness and $m$ the slope of the hquidus line. In the thermal case $\Delta=\left(T_{\mathrm{m}}-T_{\infty}\right) /(L / c), d_{c}=\gamma T_{\mathrm{m}} c / L^{2}$ and $w=\beta L / c$. One sees immediately that in both cases $d_{\mathrm{k}}$ can be written in the general form

$$
d_{\mathrm{k}}=\frac{\gamma \beta T_{\mathrm{m}}}{L D}
$$

Equations (1) and (2) can be generalized for 3D axisymmetric growth as follows [8] :

$$
\begin{array}{r}
k_{\mathrm{E}}^{2} V(1-U) \cos \theta-\frac{\left(K_{1}+K_{2}\right) U^{2}}{\left[1+U\left(1 / k_{\mathrm{E}}-1\right)\right]}-K_{\mathrm{I}} \sin \theta \frac{\mathrm{d}}{\mathrm{d} \theta}\left(\frac{U^{2}}{\cos \theta\left[1+U\left(1 / k_{\mathrm{E}}-1\right)\right]}\right) \\
+\frac{K}{V R} \frac{\mathrm{d}}{\mathrm{d} \theta}\left(\frac{R K_{1} U}{\cos \theta\left[1+U\left(1 / k_{\mathrm{E}}-1\right)\right]} \frac{\mathrm{d} U}{\mathrm{~d} \theta}\right)=0
\end{array}
$$




$$
U=\Delta-d_{h}\left(K_{1}+K_{2}\right)-V \cos \theta
$$

where $K_{1}(\theta)=\mathrm{d} \theta / \mathrm{d} s$ and $K_{2}(\theta)=|\sin \theta| / R(\theta)$ are the two principal curvatures and $R(\theta)=\left|\int_{0} \mathrm{~d} t(\cos t) / K_{1}(t)\right|$ is the radius of the circular section normal to the $z$-axis (growth direction).

Let us now discuss the solutions to equations (1) and (2) in the limiting case $d_{\mathrm{k}}=0$ corresponding to zero surface tension (2D case). To simplify, we further assume that $\Delta \ll 1$ and $k_{E}=1$. In this limit, the BLM is not strictly valud but it gives qualitatively the right physics as shown by Langer [10]. We can now substitute $U$ by $\Delta$ in all terms of equation (1) besides $d \mathrm{U} / d \theta=\mathrm{V} \sin \theta$ in the last term of equation (1). We thus obtain

$$
\frac{\mathrm{d} k}{\mathrm{~d} \theta}=\frac{k-k^{2}-\nu \cos ^{3} \theta}{k \sin \theta \cos \theta}
$$

where $k=K / \Delta, \nu=V / \Delta^{3}$. The problem now is to determine whether this equation has symmetric needle-like solutions corresponding to an even function $k(\theta)$ which starts at $\theta=0$ with a finite curvature $k_{0}$ and which tends to $k=0$ when $\theta \rightarrow \pi / 2$. Equation (4) has a singular point at $\theta=0$. Thus, for any physical solution, the numerator of the r.h.s. of equation (4) must also vanish at $\theta=0$. This regularity condition gives a relation between velocity $\nu$ and tip curvature $k_{0}$ :

$$
\nu=k_{0}-k_{0}^{2}
$$

We can expand the curvature $k(\theta)$ close to $\theta=0$ in the following form :

$$
\begin{array}{ll}
k(\theta)=\sum_{n=0} \sum_{m=0} C_{n m}\left(\theta^{2}\right)^{\alpha n+m} & \text { for } \alpha \neq 1\left(\text { or } k_{0} \neq 1 / 4\right) \\
k(\theta)=1 / 4+\sum_{n=1} \sum_{m=0}^{n} C_{n, m}^{*} \theta^{2 n}(\ln |\theta|)^{m} & \text { for } \quad k_{0}=1 / 4
\end{array}
$$

with $C_{0.0}=k_{0}$. Insertıng (6a) into (4) and equatıng coefficients of $\left(\theta^{2}\right)^{\alpha n+m}$. we find

$$
\alpha=1 / 2 k_{0}-1
$$

and recursion relations which define coefficients $C_{n, m}$ in terms of $C_{01}=3 \nu /\left[2\left(4 k_{0}-1\right)\right]$ and of the arbitrary coefficient $C \equiv C_{10}$. Constant $C$ must be evaluated by integrating equation (4) with boundary condition $k=0$ at $\theta=\pi / 2$. For example, for $\alpha=1 / 2$ (or $k_{0}=1 / 3$ ), we find $C \approx-0.39$. The same procedure can be used when $k_{0}=1 / 4$ for calculating $C_{n, m}^{*}$ in terms of $C_{1}^{*}{ }_{1}=3 \nu / 2 k_{0}=9 / 8$ and of the arbitrary constant $C^{*} \equiv C_{1}^{*}{ }_{0}$ which again must be calculated by integration.

Relations (5-7) define a contınuous set of needle-like solutions which can be parametrized by $\alpha$. The only limitation is $\alpha>0$ (to avold divergence of the curvature $k(\theta)$ at $\theta=0)$. Note that for $0<\alpha<1 / 2$, we have $\left|k^{\prime}(\theta=0)\right|=\infty$, whereas for $\alpha>1 / 2$, we have $k^{\prime}(\theta=0)=0$.

The main difference between these solutions and Ivantsov's solutions [1] is that the tip curvature and velocity now have upper limits which come from the $k_{0}^{2}$ term in equation (5) (Ivantsov's solution corresponds to $\nu=k_{0}$ in the BLM approximation). Indeed, equation (5) imposes $k_{0}<1$ and $\nu<1 / 4$ while condition $\alpha>0$ gives and additional stronger limitation $k_{0}<1 / 2$ and $\nu<1 / 4$. The dimensional velocity and tip curvature are given by

$$
v=k_{0}\left(1-k_{0}\right) w \Delta^{3}, \quad \kappa_{0}=k_{0}(w / D) \Delta, \quad k_{0}<1 / 2 .
$$


The problem now is to understand what happens when surface tension and anisotropy are included. In this case equations (2) and (4) are generalized as follows:

$$
\begin{gathered}
U=\Delta-K d_{\mathrm{k}}\left(1-\gamma_{m} \cos m \theta\right)-V \cos \theta\left(1-\beta_{m} \cos m \theta\right) \\
\nu \cos ^{3} \theta-k-\frac{k}{\nu} \cos ^{2} \theta \frac{\mathrm{d}}{\mathrm{d} \theta}\left\{\frac { k } { \operatorname { c o s } \theta } \frac { \mathrm { d } } { \mathrm { d } \theta } \left[k \delta\left(1-\gamma_{m} \cos m \theta\right)+\right.\right. \\
\left.\left.+\nu \cos \theta\left(1-\beta_{m} \cos m \theta\right)\right]\right\}=0
\end{gathered}
$$

where $\delta=d_{\mathrm{k}} / \Delta^{2} . \gamma_{m}$ is the surface stiffness anisotropy while $\beta_{m}$ is the anisotropy of the kinetic coefficient $\beta$ for $m$-fold symmetry. We also assume that directions of minımal surface stıffness and of maximal kinetic coefficient coincide with the growth direction.

In the simplest case, we include only kinetic anisotropy (with zero surface tension, $\delta=0$ ). Thus, for sufficiently small anisotropy $\beta_{m}$, there exists a continuous set of solutions given by:

$$
\nu=k_{0}-k_{0}^{2} / B, \quad \alpha=\left(B / 2 k_{0}-1\right)>0
$$

with $B=1 /\left[1-\beta_{m}\left(m^{2}+1\right)\right]$ and lımiting values of velocity and tip curvature given by $\nu<B / 4$ and $k_{0}<B / 2$. The solutions disappear when $\beta_{m}\left(m^{2}+1\right) \geqslant 1$ (Fig. 1 ).

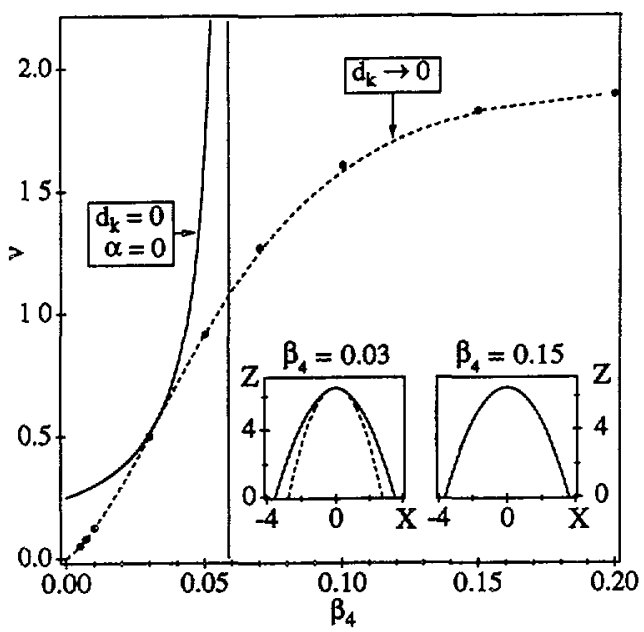

Fig. 1. - Dimensionless velocity as a function of kinetic anisotropy. The solid line gives the maximal velocity of « kinetıc dendrites ». Dots correspond to velocities $\nu_{0}$ of dendrites selected by anisotropy (see also inset in Fig. 2). Two dendrite profiles (with the unit of length $D /(w . \Delta . \nu)$ ) corresponding to two different values of $\beta_{4}$ and $\delta=10^{-3}$ are given in the insets. The profiles are compared with the parabolic profiles having the same tip curvature (dashed lines; in the right inset the two profiles are indistinguishable on the choosen scale).

When we include isotropic surface tension without any anisotropy $\left(\gamma_{m}=\beta_{m}=0\right)$ all solutions disappear as usual [10]. This result is obtained from numerics and reflects the impossibility of finding physical solutions $k(\theta)$ starting from $\theta=\pi / 2$ with $k=0$ and arriving at $\theta=0$ with $k^{\prime}(0)=0$ [11] (in all cases, $k^{\prime}(0)$ is found to be negative). 
When anisotropy is included, we find numerically some values of $\nu$ which provide $k^{\prime}(0)=0$, thus giving a discrete spectrum of solutions for each given value of $\delta$ [12]. In figure 2, we plot maximum selected velocity $\nu$ as a function of dimensionless parameter $\delta=d_{\mathrm{k}} / \Delta^{2}$. These curves have been numerically calculated for different values of $\beta_{4}$ and $\gamma_{4}$. Two different cases can be considered depending on the existence or not of surface stiffness anisotropy :

- if $\gamma_{4} \neq 0$, there are two asymptotic regimes : in the capillary regime, at $\delta \rightarrow \infty$, the dimensionless velocity $\nu$ scales like $\gamma_{4}^{7 / 4} / \delta$ in agreement with the calculations of Langer and Hong for the BLM [9]. In the kinetic regime, at $\delta \rightarrow 0$ (or $d_{\mathrm{k}} \rightarrow 0$ ), velocity $\nu$ tends to a limit $\nu_{0}$ (which is obtained by extrapolation) depending only on $\beta_{4}$ (see inset in Fig. 2). At small anisotropy $\nu_{0} \sim \beta_{4}^{125}$, in agreement with a previous analytical prediction [7]. This selected velocity is plotted again in figure 1 in order to compare it with the maximal velocity of « kinetic » dendrites corresponding to $d_{\mathrm{k}}=0$. Two points must be emphasızed : first, the selected dendrite still exists when $\beta_{4}>1 / 17$ and «kinetic » dendrites disappear. Second, when $\beta_{4} \approx(0.02-0.04)$, the selected velocity is close to the maximal velocity of « kinetic » dendrite.

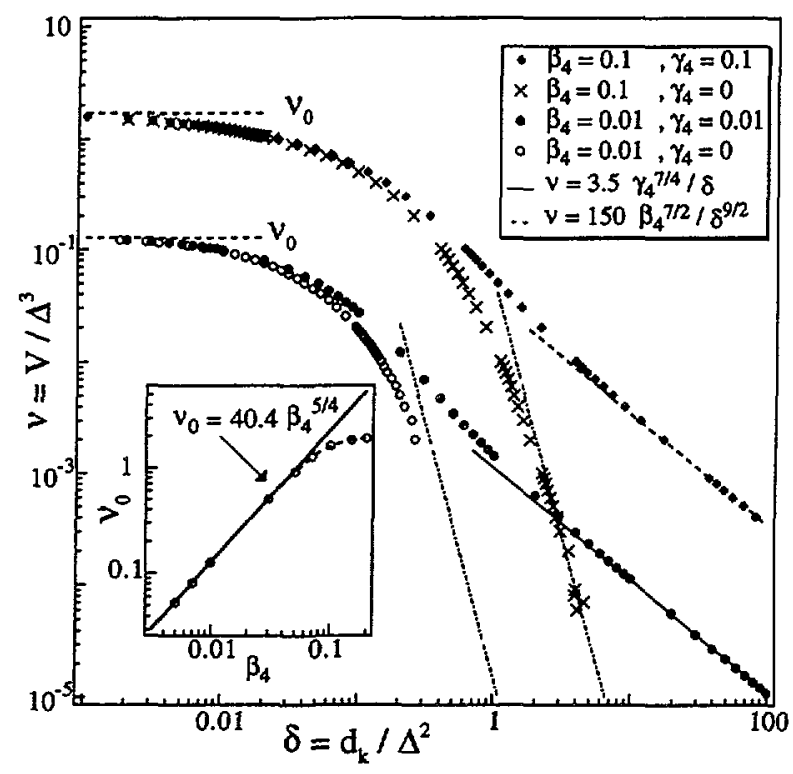

Fig. 2. - Dimensionless velocity $\nu \equiv V / \Delta^{3}$ as a function of $\delta \equiv d_{k} / \Delta^{2}$ for different values of surface stiffness anisotropy $\gamma_{4}$ and kinetic anısotropy $\beta_{4}$. The limiting velocity $\nu_{0}$ found by extrapolation to $\delta=0$ are given in the inset.

- When $\gamma_{4}=0$ two asymptotic regimes still exist as predicted by Brener $[2,13]$. The first one at $\delta \rightarrow 0$ is independent of $\gamma_{4}$ and thus coincides with the kinetic regime discussed just before, while the second one, at $\delta \rightarrow \infty$, is characterized by $\nu \sim \beta_{4}^{7 / 2} \delta^{-9 / 2}$ The latter result is in agreement with the analytical prediction of Brener. We can again emphasize that this result is easier to find with the BLM than by solving the full nonlocal problem [14].

So far, we have assumed that $\Delta \ll 1$ and $k_{\mathrm{E}}=1$. We now discuss « kinetic » dendrites for arbitrary $\Delta$ and $k_{\mathrm{E}}$ without anisotropy and surface tension. Using the same procedure as that 
used previously to derive equations (4)-(7), we find :

$$
\begin{gathered}
k_{\mathrm{E}}^{2} V(1-\Delta+V)\left[1+(\Delta-V)\left(1 / k_{\mathrm{E}}-1\right)\right]-K_{0}(\Delta-V)^{2}+K_{0}^{2}(\Delta-V)=0 \\
\alpha=\left[(\Delta-V) / 2 K_{0}-1\right]>0 .
\end{gathered}
$$

Similar equations can be obtained for 3D axisymmetrıc dendrites using equations ( $\left.1^{\prime}\right)$ and $\left(2^{\prime}\right)$ :

$$
k_{\mathrm{E}}^{2} V(1-\Delta+V)\left[1+(\Delta-V)\left(1 / k_{\mathrm{E}}-1\right)\right]-2 K_{0}(\Delta-V)^{2}+2 K_{0}^{2}(\Delta-V)=0
$$

and the same relation for $\alpha$ as that given by equation (13). In this case, the two curvatures at the tip of the dendrite are equal $\left(K_{1}(0)=K_{2}(0)=K_{0}\right)$ and the tip radius equals $1 / K_{0}$.

Equations (12-14) only give the relation between growth velocity and tip curvature as well as their upper limits (Fig. 3). On the other hand, to find dendrite profiles (see insets in Fig. 3), we need to solve numerically the corresponding differential equations (1) or (1') in $K(\theta)$ with condition $K(\pi / 2)=0$ at $\Delta<1$ (usual dendrite with a parabolic tarl) and with condition $K\left(\theta_{0}\right)=0$ at $\Delta>1$ (angular dendrite $[15,16]$ ). In the latter case, $\theta_{0}$ is given by $V \cos \theta_{0}=(\Delta-1)$.

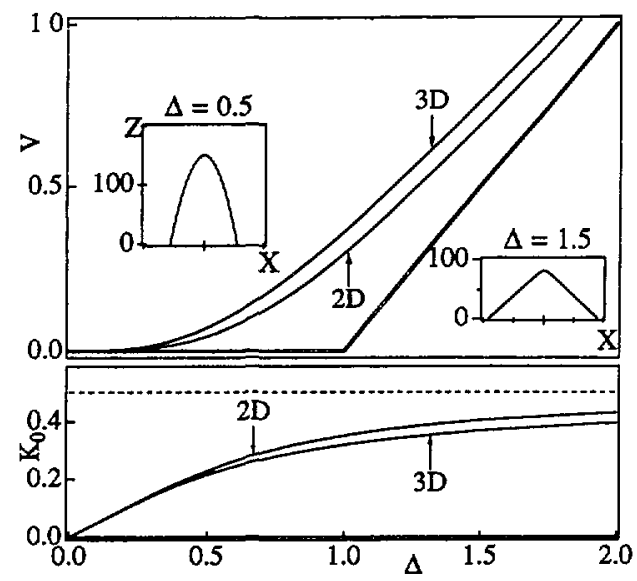

Fig. 3. - Tip velocity $V$ (in unit of $w$ ) and tip curvature $K_{0}$ (in unit $w / D$ ) versus supercooling for two and three-dimensional dendrites $\left(k_{\mathrm{E}}=1\right)$. Solid lines correspond to « kinetic » dendrites of maximal velocity $\left(d_{\mathrm{k}}=0\right.$ and $\left.\alpha \rightarrow 0\right)$. The thick solid lines: $V=0$ at $\Delta<1, V=(\Delta-1)$ for $\Delta>1$, and $K_{0}=0$ correspond to steady-state growth of the planar front. They also correspond to kinetic dendrites in the limit $\alpha \rightarrow \infty$. Two profiles for 2D « kinetic » dendrites $\left(d_{\mathrm{k}}=0\right.$ and $\left.\alpha \rightarrow 0\right)$ are shown in the insets.

In conclusion, we have found a new basic continuous family of solutions which takes into account interface kinetics but not surface tension. This solution describes a needle-like dendrite at arbitrary supercooling $\Delta$ smaller or larger than 1 . Consequently, it should be more appropriate for describing dendrites at large supercooling than the usual Ivantsov solution only valıd at $\Delta<1$. It could also serve as a startıng approximation in a more complete theory.

\section{Acknowledgments.}

This work was supported by CNES contract No. 93.0215. D. Temkin is grateful for the Poste Rouge provided by the CNRS. 


\section{References}

[1] Ivantsov G. P., Dokl. Akad. Nauk SSSR 58 (1947) 467.

[2] For a review see Brener E. A., Mel'nıkov V. I., Adv. Phys. 40 (1991) 53.

[3] Willnecker R., Herlach D. M., Feuerbacher B., Phys. Rev. Lett. 62 (1989) 2707.

[4] Glicksman M. E., Schaefer R. J., Ayers J. D., Metal Trans. A 7A (1976) 1747.

[5] Géminard J. C., Oswald P., Temkin D., Malthête J., Europhys, Lett. 22 (1993) 69.

[6] Lemieux M.-A., Liu J. and Kotl1ar G., Phys. Rev. A 36 (1987) 1849.

[7] Brener E. A., Geilikman M. B., Temkin D. E., Sov. Phys. JETP 67 (1988) 1002.

[8] Ben-Jacob E., Goldenfeld N., Langer J. S., Schön G., Phys. Rev. A 29 (1984) 330.

[9] Langer J. S., Hong D. C., Phys. Rev. A 34 (1986) 1462.

[10] Langer J. S., Phys. Rev. A 33 (1986) 435 and also Ref. [8].

[11] Condition $k^{\prime}(0)=0$ comes from symmetry and from the fact that the differential equation is now of second order (see Refs. [8-10]).

[12] Ben-Jacob E., Garik P., Mueller T. and Grier D., Phys. Rev. A 38 (1988) 1370.

[13] Brener E. A., J. Cryst. Growth 99 (1990) 165.

[14] There is nevertheless a restriction to this conclusion concerning the scalng with $\Delta$. Indeed, in the limit $\Delta \rightarrow 0$, exact scaling for dimensional velocity and tip curvature reads $v \sim \gamma_{4}^{7 / 4}\left(D / d_{c}\right) \Delta^{4}$ and $\mathcal{K}_{0} \sim \gamma_{4}^{7 / 4}\left(1 / d_{\mathrm{c}}\right) \Delta^{2}$ [2] whereas BLM gives $v \sim \gamma_{4}^{7 / 4}\left(D / d_{\mathrm{c}}\right) \Delta^{5}$ and $\kappa_{0} \sim \gamma_{4}^{7 / 4}\left(1 / d_{\mathrm{c}}\right) \Delta^{3}$. We suggest that this difference in the power of $\Delta$ exists in all the asymptotic regimes we have discussed.

[15] Brener E. A., Temkin D. E., Europhys. Lett. 10 (1989) 171.

[16] Classen A., Misbah C., Miiller-Krumbhaar H., Saito Y., Phys. Rev. A 43 (1991) 6920. 